\title{
Connection between the Periodontal Disease and Diabetes Mellitus
}

\author{
Ambarkova Vesna* \\ Department for preventive and Pediatric dentistry, University Ss Cyril \& Methodius, Macedonia \\ *Corresponding author: Ambarkova Vesna, Department of Paediatric and Preventive Dentistry, Faculty of Dental Medicine, University St Cyril and \\ Methodius, Macedonia
}

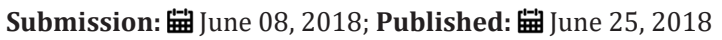

\section{Short Communication}

Diabetes Mellitus is a metabolic disorder affecting more than 16 million people worldwide and is characterized by hyperglycemia resulting from a defect in insulin secretion, insulin resistance, or both. Type 1 Diabetes Mellitus is a result of the destruction of beta cells in the pancreatic islets or islets of Langerhans probably because of an autoimmune or viral disease, which leads to a lack of insulin in the body. Type 2 Diabetes Mellitus is characterized by a lack of response to insulin by target cells, although the production of insulin is usually normal or even increased in these individuals, and is due to a change in the structure or number of cellular insulin receptors. It is thought that type $2 \mathrm{DM}$ may be a congenital disorder of the immune system and a result of a chronic, low level of inflammatory process. The number of adults diagnosed with DM type 2 in the world is expected to increase from 135 million in 1995 to about 300 million in 2025. People with type 2 diabetes mellitus covers $90 \%$ of the population with Diabetes Mellitus [1].

A recent hypothesis involves subclinical chronic inflammation with insulin resistance, prompting the development of DM type 2. A trigger of inflammation potentially involves an oral infection, which can lead to a cascade of events including an increase in cytokine production, activation of the proteins synthesis in the acute-phase leading to a reduction in pancreatic B-cell function, apoptosis, and insulin resistance, or chronic subclinical inflammation is associated with insulin resistance and initiation of Diabetes Mellitus type 2 [2]. Diseases that lead to chronic inflammatory response increase the risk of Diabetes Mellitus development and affect DM control and the development of Diabetes Mellitus complications.

The diabetes mellitus and periodontal disease are complex chronic diseases with established two-way linkage. In patients with $\mathrm{DM}$, there is a direct dose-dependent relationship between the severity of periodontal disease and diabetes mellitus complications. Periodontal disease is the 6th most common complication of diabetes mellitus. There are studies showing that poor control of glycemia can lead to poor oral health, and these individuals are 2.8 times more likely to develop periodontal disease and a 4.2-times more chance for development of the bone destruction. There is consistent evidence that the severe form of periodontal disease affects poorly the levels of glycemia and HbA1C (hemoglobin $\mathrm{A} 1 \mathrm{C}$ ) in patients with and without diabetes mellitus [3].

Studies that examined the relative prevalence of parodontal pathogens between individuals with diabetes mellitus type 1 and type 2 did not receive a statistically significant correlation except for A. Actinomycetemcomitans [4]. Once parodonto-pathogenic bacteria settle in a diabetic host, periodontal infection may exacerbate micro-vascular complications of diabetes mellitus, and further lead to macro-vascular complications.

One probable biological mechanism of why diabetics have a more severe periodontal disease form is that blood hyperglycaemia causes advanced glycation end-products (AGE) whose accumulation affects the migration and phagocytic activity of phagocytes, resulting in the formation of a more pathogenic sub-gingival flora. The gradual transformation of the sub-gingival microflora into the gram-negative flora will be a chronic source of systemic challenge with an "infection-traced" cytokine upward regulation. The periodontal disease can and itself lead to insulin resistance, contributing to the cycle of hyperglycaemia, AGE protein accumulation, extending the classical pathway to diabetic degradation and destruction of connective tissue. Hence, the relationship between diabetes mellitus and periodontal disease becomes two-way. Both diseases have a relatively high incidence in the population and are polygenic disorders in which there is some degree of dysfunction of the immune system [5].

Recently, Blauer et al. also investigated whether plasma concentrations of inflammatory markers were related to obesity, insulin sensitivity, and hyperglycaemia, and obtained significant correlation [2]. In a similar study, the Augsburg study, the authors concluded that in patients with DM type 2 the levels of IL-18, CRP and IL-6 [6] were elevated. These observations indicate that the immune response to the acute phase is associated with insulin resistance, and may be the thread of the development of DM type 2. Randomized controlled trials have shown that the mechanical periodontal terrarium leads to a $0.4 \%$ reduction in $\mathrm{HbA} 1 \mathrm{C}$ levels 
within three months, which is equivalent to introducing a second drug in the therapy of the patient with diabetes mellitus [7].

A study suggests that effective therapy of the periodontal disease in patients with diabetes mellitus can reduce the level of AGE serum products that cause hyperglycaemia, and thus parodontal treatment affects glycemic control [8]. The therapy of the periodontal disease must be considered as an integral part of diabetes mellitus treatment and control. Major efforts should be aimed at preventing periodontal disease in patients at risk of diabetes mellitus, as well as in those patients with poor metabolic control.

\section{References}

1. King H, Aubert RE, Herman WH (1998) Global burden of diabetes, 19952025: prevalence, numerical estimates, and projections. Diabetes Care 21(9): 1414-1431.

2. Bluher M, Fasshauer M, Tonjes A, Kratzsch J, Schon MR, et al. (2005) Association of interleukin-6, c-reactive protein, interleukin-10 and ad- iponectin plasma concentrations with measures of obesity, insulin sensitivity and glucose metabolism. Exp Clin Endocrinol Diabetes 113(9): 534-537.

3. Matthews DC (2002) The relationship between diabetes and periodontal disease. J Can Dent Assoc 68(3): 161-164.

4. Tervonen T, Oliver RC, Wolff LF, Bereuter J, Anderson LA (1994) Prevalence of periodontal pathogens with varying metabolic control of diabetes mellitus. J Clin Periodontol 21(6): 375-379.

5. Anil S, Remani P, Ankathil R, Vijayakumar T (1990) Circulating Immune complexes in diabetic patients with periodontitis. Ann Dent 49(2): 3-5.

6. Genco RJ, Grossi SG, Ho A, Nishimura F, Murayama Y (2005) A proposed model linking inflammation to obesity, diabetes, and periodontal infections. J Periodontol 76(suppl 11): 2075-2084.

7. Stewart JE, Wager KA, Friedlander AH, Zadeh HH (2001) The effect of periodontal treatment on glycemic control in patients with type 2 diabetes mellitus. J Clin Periodontol 28(4): 306-310.

8. Grossi SG, Genco RJ (1998) Periodontal disease and diabetes mellitus: A two-way relationship. Ann Periodonto 3(1): 51-61.
Creative Commons Attribution 4.0

International License

For possible submissions Click Here

\section{Submit Article}

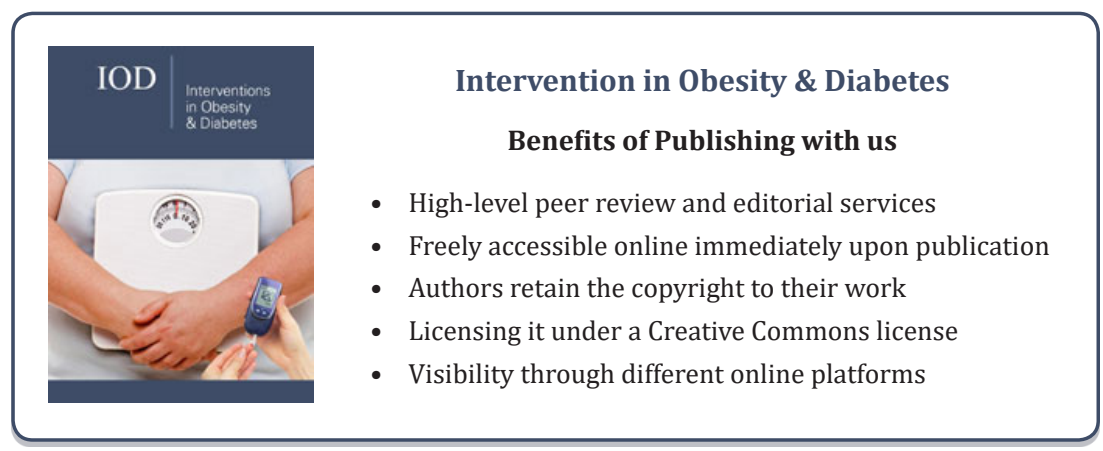

